\title{
Thermal Spray Forming of Heat Transfer Devices
}

\author{
Sanjeev Chandra \\ Department of Mechanical \& Industrial Engineering, Faculty of Applied Science \& Engineering \\ University of Toronto \\ Toronto, Canada
}

Thermal spray deposition refers to a family of processes in which a coating material (metal, ceramic or polymer) is introduced as a powder or wire into a hot, high velocity gas jet where it melts and atomizes. The small droplets produced are propelled onto a surface where they flatten, coalesce and form a dense coating that adheres strongly to the substrate. Thermal spray is widely used to apply protective coatings on components exposed to heat, wear and corrosion, but it can also be used an additive manufacturing technique to make devices for thermal management. Thermal spray is a relatively low-cost, highdeposition rate process that is environmentally friendly since it produces no hazardous emissions. Compact, high efficiency heat exchangers have been made by spraying metal skins on the exterior surfaces of metal foams that have a large internal surface area for heat transfer. Composite heat sinks have been made by spraying thin metal layers on polymer scaffoldings, producing lightweight devices that use only a minimal amount of metal where required. It is possible to spray porous metal layers by reducing the velocity and temperature of impacting spray particles so that they do not coalesce completely with each other. Such porous layers can be used as wicks for capillary transport of liquids in heat pipes and heat spreaders. By spraying through masks it is possible to make channels in the wicks to maximize their permeability and the rate of liquid flow. Spraying allows coatings to be applied rapidly over a large area, making it feasible to make thermal management devices that are very large in extent. 